Erratum

\title{
Expression of DC8 is associated with, but not dependent on embryogenesis
}

\author{
Jin-Chen Cheng, Kevin Andrew Seeley, Pascale Goupi ${ }^{1}$ and Zimmay Renee Sung* \\ Department of Plant Biology, 111 Koshland Hall, University of California Berkeley, CA 94720, USA \\ (*author for correspondence); ${ }^{1}$ Current Address: Physiologie de la Differentiation et Biotechnologies Végétales, \\ Université des Sciences et Technologies de Lille Flandres Artois, Bat. SN2 59655 Villeneuve d'Asco \\ Cedex, France \\ Plant Molecular Biology 31: 127-141, 1996.
}

Typography of the authors should be printed as above. This work was carried out entirely at UC Berkeley, USA. On page 129 (right column, line 22) p87P1.7 should read p8P1.7. 